\title{
Caracterización clinicoepidemiológica de pacientes especiales con gingivitis crónica
}

\section{Clinical epidemiological characterization of special patients with chronic gingivitis}

Caracterização clínica e epidemiológica de pacientes especiais com gengivite crônica

Juan A. Oliveira del Rio I

juanodelrio@gmail.com

Zully A. Macías-Velásquez ${ }^{\text {II }}$

zyllyma@gmail.com

Alba M. Mendoza-Castro
albammendoza@gmail.com

Recibido: 30 de enero de 2017 * Corregido: 20 de febrero de $2017 *$ Aceptado: 20 junio de 2017

I. Docente, Universidad Laica Eloy Alfaro de Manabí, Manta, Ecuador.
II. Docente, Universidad Laica Eloy Alfaro de Manabí, Manta, Ecuador.
III. Docente, Universidad Laica Eloy Alfaro de Manabí, Manta, Ecuador. 


\section{Resumen}

Se realizó una investigación cuantitativa -cualitativa descriptivo y transversal en un total de 40 pacientes especiales con gingivitis crónica, atendidos en la consulta odontológica desde enero de 2016 hasta enero de 2017, con la finalidad de caracterizarles según variables clínicas y epidemiológicas: edad, sexo, factores de riesgo, grado de inflamación y nivel de conocimiento. Entre los resultados principales prevalecieron el sexo masculino (62.5\%), el grupo etario de 15-19 años $(60.0 \%)$, así como la higiene bucal deficiente $(80.0 \%)$ como factor de riesgo; entre estos últimos, la mayoría presentaron gingivitis crónica edematosa $(37.2 \%)$ e inflamación moderada $(55.5 \%)$ y severa $35.5 \%$. El nivel de conocimiento sobre la patología por parte de los padres fue inapropiado. La práctica inadecuada del cepillado dental, más la dieta blanda dada por la incapacidad intelectual de este tipo de paciente, favoreció la aparición de esta enfermedad.

Palabras clave: Paciente especial, enfermedad periodontal, gingivitis crónica. 


\begin{abstract}
A quantitative-descriptive and cross-sectional investigation was carried out in a total of 40 special patients with chronic gingivitis, attended in the dental office from January 2016 to January 2017, in order to characterize them according to clinical and epidemiological variables: age, sex, risk factors, degree of inflammation and level of knowledge. Among the main results, the male sex prevailed $(62.5 \%)$, the age group of $15-19$ years $(60.0 \%)$, as well as the deficient oral hygiene $(80.0 \%)$ as a risk factor; among the latter, the majority had chronic edematous gingivitis $(37.2 \%)$ and moderate inflammation $(55.5 \%)$ and severe $35.5 \%$. The level of knowledge about the pathology by the parents was inappropriate. The inadequate practice of tooth brushing, plus the soft diet given by the intellectual incapacity of this type of patient, favored the appearance of this disease.
\end{abstract}

Key words: Special patient, periodontal disease, chronic gingivitis. 


\section{Introducción.}

La atención odontológica al paciente discapacitado va cobrando cada día más interés, en los profesionales de la salud; principalmente en aquellos que tienen una concepción integral de la misma. ${ }^{1}$

Para los odontólogos, un paciente especial es aquel que presenta signos y síntomas que lo alejan de la normalidad, sean de orden físico, mental o sensorial; así como del comportamiento y que, para su atención odontológica exige maniobras, equipamientos y personal de ayuda especial, con capacidad de atender las necesidades que éstos generan, escapando de los programas y rutinas estándares que se llevan a cabo para el mantenimiento de la salud bucodental de la población. ${ }^{2}$

Según la OMS, dos terceras partes de la población, con discapacidad, no reciben atención bucodental alguna. La OMS define la discapacidad como un término genérico que incluye deficiencias de las funciones y estructuras corporales, limitaciones en la actividad y restricciones en la participación e integración de un individuo y su entorno. ${ }^{3}$

Una deficiencia puede deberse a una enfermedad congénita, una alteración del desarrollo, proceso degenerativo como el Alzheimer o el Parkinson, enfermedades graves o accidentes; éstos generan una disminución o ausencia de capacidad dentro de los límites normales, que colocan al individuo en una situación de desventaja social. ${ }^{3}$

La discapacidad es un problema de salud pública que afecta no sólo al individuo que la padece, sino también a su entorno familiar; se calcula que por cada persona con discapacidad, al menos cuatro individuos más se verán directamente implicados en el problema. Esto se relaciona además con las alteraciones médicas, con limitaciones sociales que se originen y que traen como 
consecuencia otro problema aún más grave; la discriminación. Ésta se entiende como exclusión, limitación o preferencia basada en la raza, sexo, idioma, religión y opiniones políticas; origen social, posición económica o características físicas que tengan como fin, disminuir la igualdad en el trato. ${ }^{3}$ Las personas con discapacidad sufren de mala salud, su nivel educativo es más bajo, tienen menos oportunidades económicas y mayores tasas de pobreza que las personas sin discapacidad. Esto se debe en gran parte a la falta de servicios disponibles para tratarlos y a los diversos obstáculos a que se enfrentan en su vida cotidiana

Los problemas buco dentales de la humanidad se remontan a tiempos muy lejanos por lo que se puede afirmar que han sido compañeros inseparables de nuestros antepasados desde hace millones de años.

La enfermedad periodontal y los trastornos bucofaciales de otra índole constituyen problemas singulares en la asistencia médica y odontológica de personas con discapacidad. Estas enfermedades suelen ser crónicas y por ello complican durante toda la vida a otros problemas mentales o físicos incapacitantes. ${ }^{4}$

Las periodontopatías son consideradas un conjunto de alteraciones en la fisiología de los tejidos que rodean y soportan los dientes. Es una entidad indolora que puede asumir varias formas, presentando diversos grados de severidad que serán resultado de la virulencia, cantidad y frecuencia de las agresiones ambientales locales, modificadas por la resistencia y los factores reparadores; interviniendo otros factores sistémicos que son capaces de modificar la respuesta a los irritantes locales y afectan directamente la micro vascularización de la encía. ${ }^{5}$ 
Se pueden categorizar como gingivitis: que es la inflamación de la encía, con un cambio de coloración a rojizo, de aspecto liso y brillante, y consistencia blanda; y a periodontitis: que es la inflamación gingival acompañada de pérdida de la inserción de tejido conectivo. La gingivitis es considerada una forma inicial de la enfermedad periodontal, puede convertirse en una periodontitis, incrementando su severidad en base a la combinación de factores como la falta de cepillado. ${ }^{6}$

En el ecuador se trabaja por el desarrollo de procesos de promoción de la salud y el fortalecimiento del Sistema Nacional de Salud para la prevención y control de las enfermedades no transmisibles, incluye también las discapacidades y la salud bucal, reduciendo la carga sanitaria y social de estas enfermedades y/o problemas de salud. ${ }^{7}$

\section{Metodología.}

Se realizó una investigación cuantitativa -cualitativa descriptivo y transversal de 40 pacientes especiales con gingivitis crónica, atendidos en la consulta odontológica desde enero de 2016 hasta enero de 2017, con la finalidad de caracterizarles clinicoepidemiologicamente.

Se asumieron para la investigación variable como: edad, sexo, factores de riesgo, grado de inflamación y nivel de conocimiento.

Paciente especial es el niño, joven o adulto que presenta manifestaciones clínicas que lo alejan de la normalidad, con algún tipo de discapacidad física, psíquica, sensorial o problemas del comportamiento.

En este grupo se consideraron los retrasados mentales (leves y moderados) según diagnóstico de historia clínica que presentaban los padres. 
La evaluación del conocimiento sobre salud oral de los padres se realizó mediante un cuestionario: satisfactorio y no satisfactorio

Una vez recogida la información, se procesó a través del programa estadístico SPSS, versión 15,0. Se utilizaron como medidas de resumen la frecuencia absoluta y el porcentaje.

Antes de incluir a los pacientes en el estudio se les informó los objetivos de este y se solicitó su consentimiento informado.

\section{Resultados.}

En la tabla 1 se observa que de los 40 pacientes examinados existe predominio del sexo masculino con $62.5 \%$ en relación con el femenino. Con respecto al grupo de edad se puede constatar que el de 15-19 años fue el más numeroso con un valor del 60.0\% de los afectados, seguido del de 5-9 años con el $20 \%$ respectivamente

Tabla 1 Pacientes especiales con gingivitis crónicas.

\begin{tabular}{|c|c|c|c|c|c|c|}
\hline $\begin{array}{l}\text { Grupo de } \\
\text { edades en } \\
\text { años }\end{array}$ & \multicolumn{3}{|c|}{ Sexo } & \multicolumn{2}{c|}{ Total } \\
& Femenino & \multicolumn{3}{c|}{ Masculino } \\
\cline { 2 - 7 } & No & $\%$ & No & $\%$ & No & \% \\
\hline $5-9$ & 2 & 13.3 & 6 & 24.0 & 8 & 20 \\
\hline $10-14$ & 2 & 13.3 & 3 & 12.0 & 5 & 12.5 \\
\hline $15-19$ & 10 & 66.6 & 14 & 56.0 & 24 & 60.0 \\
\hline $19+$ & 1 & 6.6 & 2 & 8.0 & 3 & 7.5 \\
\hline Total & 15 & 37.5 & 25 & 62.5 & 40 & 100 \\
\hline
\end{tabular}

La higiene bucal deficiente sobresalió como el factor de riesgo más frecuente en la población estudiada según se observa en la tabla 2, pues el $80.0 \%$ de los pacientes presentaban este factor; en orden de frecuencia siguieron el apiñamiento dentario $45.0 \%$ el consumo de 
medicamentos $(20.0 \%)$ y el empaquetamiento de alimento con un $17.5 \%$ respectivamente. Se debe destacar que de los 3 pacientes examinados de más de 19 años fumaban.

Los pacientes con higiene bucal deficientes (32), 12 padecían de gingivitis crónica edematosa $(37.2 \%)$,el resto presentaron gingivitis fibroedematosa

Tabla 2 Pacientes especiales con gingivitis crónicas según factores de riesgo.

\begin{tabular}{|l|c|c|c|c|}
\hline \multicolumn{1}{|c|}{$\begin{array}{c}\text { Factores de } \\
\text { riesgo }\end{array}$} & \multicolumn{2}{|c|}{ No } & \multicolumn{2}{c|}{ No } \\
\hline $\begin{array}{l}\text { Higiene bucal } \\
\text { deficiente }\end{array}$ & 32 & 80.0 & 8 & 20 \\
\hline $\begin{array}{l}\text { Apiñamiento } \\
\text { dentario }\end{array}$ & 18 & 45.0 & 10 & 25 \\
\hline $\begin{array}{l}\text { Consumo de } \\
\text { medicamento }\end{array}$ & 8 & 20 & 4 & 10 \\
\hline $\begin{array}{l}\text { Empaquetamiento } \\
\text { de alimentos }\end{array}$ & 7 & 17.5 & 3 & 7.5 \\
\hline Tabaquismo & 1 & 2.5 & 0 & 0 \\
\hline
\end{tabular}

Se observa en la tabla 3 del total de pacientes examinados predomino la inflamación moderada con un $55.5 \%$ prevaleciendo en el sexo masculino. En el sexo femenino también prevaleció este tipo de inflamación. Existió un porciento considerable de pacientes con inflamación severa con $35.5 \%$ 
Tabla 3 Pacientes especiales según el grado de inflamación de la gingivitis crónica.

\begin{tabular}{|l|c|c|c|c|c|c|}
\hline \multirow{2}{*}{$\begin{array}{c}\text { Grado de } \\
\text { inflamación }\end{array}$} & \multicolumn{3}{c|}{ Sexo } & \multicolumn{2}{c|}{ Total } \\
& Femenino & \multicolumn{3}{c|}{ Masculino } & \\
\cline { 2 - 7 } & No & $\%$ & No & $\%$ & No & $\%$ \\
\hline Leve & 3 & 20 & 1 & 4 & 4 & 10 \\
\hline Moderada & 7 & 46.6 & 15 & 60 & 22 & 55.5 \\
\hline Severa & 5 & 33.3 & 9 & 36 & 14 & 35.0 \\
\hline Total & 15 & 37.5 & 25 & 62.5 & 40 & 100 \\
\hline
\end{tabular}

Al evaluar el nivel de conocimiento que tenían los padres con respecto a la enfermedad gingivitis crónica solamente 9 padres evidenciaron un conocimiento apropiado represento $22.5 \%$ (tabla 4 )

Tabla 4. Nivel de conocimiento de salud sobre gingivitis y salud bucal de los padres

\begin{tabular}{|l|c|c|}
\hline Nivel de conocimiento & No & \% \\
\hline Satisfecho & 9 & 22.5 \\
\hline No satisfecho & 31 & 77.5 \\
\hline Total & 40 & 100 \\
\hline
\end{tabular}

\section{Discusión.}

En cuanto a la distribución por sexo, los resultados de esta casuística concuerdan con los de Sueiro et al, ${ }^{8}$ y Zamora Tejeda ${ }^{9}$ quienes también encontraron predominio del masculino.

Muchos científicos han dedicado miles de horas al estudio de los factores causales. La periodoncia moderna enfoca estos problemas bajo un prisma nuevo, el de los llamados factores de riesgo, para el inicio y progresión de la enfermedad periodontal. ${ }^{10}$ En concordancia con esto último, en esta casuística predominaron, por un lado, la higiene bucal deficiente, debido a que la discapacidad intelectual de estos niños no les permite practicar correctamente el cepillado dental; por otro, el apiñamiento dentario, el cual favorece el avance y aparición de gingivitis crónica. 
Autores $^{10-9}$ en estudios similares señalan un mayor porcentaje de niños con higiene bucal entre regular y mala, lo que conlleva a un pobre estado de salud gingival. En cuanto al apiñamiento dentario, los resultados del presente estudio son similares a los de Valerazo y Saritama, ${ }^{11}$ quienes señalan que aunque este factor de riesgo se presentó en menor frecuencia, es actualmente el tipo de maloclusion más común y tiene un alto grado de variabilidad, atribuido a factores como evolución, genética y color de la piel, entre otros; hallazgo que también concuerda con lo referido por Zaldívar et al. $^{12}$

La gingivitis es un proceso inflamatorio de las encías, de carácter reversible, sin migración apical del epitelio de inserción, que no altera sustancialmente el tejido de soporte del diente. ${ }^{13}$

La inflamación moderada de los tejidos gingivales fue superior en el sexo masculino al presentarse la mayor cantidad de pacientes con higiene bucal deficiente; resultado que coincide con lo observado por Zaldívar et $a l^{12}$ en un estudio similar

Los padres desempeñan un papel crucial en el soporte emocional, necesidad de adaptación comportamental frente a enfermedades o tratamientos y en el financiamiento de los costos en la salud de los niños. Para que haya entonces salud bucal, los padres deben ser educados y concientizados para sensibilizar tempranamente a sus hijos en la adquisición de comportamientos que permitan un buen desenvolvimiento de hábitos de salud bucal. ${ }^{14}$

Las acciones para proteger la salud bucal de los niños se basan en una constante participación de los padres o tutores, en programas del área materno-infantil y al trabajo de pediatría de una manera general. Sin embargo, existe una gran dificultad para conseguir la colaboración de los padres, para la atención o realización de maniobras preventivas en el ámbito doméstico. ${ }^{14}$ 
En esta serie el nivel de conocimientos de los padres acerca de la salud bucal fue no satisfactorio en un mayor por ciento. En ese sentido, en el estudio llevado a cabo por Chileno Arana $\mathrm{M},{ }^{15}$ los resultados han evidenciado un deficiente conocimiento de los padres sobre salud oral y una higiene oral mala en los escolares.

Teniendo en cuenta todo lo anterior se concluye una frecuencia elevada de pacientes especiales con gingivitis crónica, principalmente del sexo masculino y del grupo etario de 15-19 años; predominando la higiene bucal deficiente, y la gingivitis crónica edematosa, con un grado de inflamación gingival moderada

\section{Referencias bibliográficas.}

1. Pereira S. Discrasias sanguíneas. Consideraciones generales y Manejo Odontológico. Universidad del Zulia. Facultad de Odontología. Cátedra de Medicina Interna 2003; 185-63.

2. Pérez Requejo JL. Hematología Tomo II. $3^{\text {a }}$ Edición. Ed. Disinlilmed, C.A. Caracas

3. Marchena Rodríguez, L, Fernández Ortega, C M. Atención odontológica a pacientes discapacitados. Revistas europea de odontoestomatologia 2015. [citado 12 oct 2017]. Disponible en : http://www.redoe.com/ver.php?id=219

4. Bécquer Águila BJ, López Díaz Z. Alteraciones de la salud del adulto mayor. En: González Naya G, Montero del Castillo ME, Aldama Bellon Y, Alfaro Mon M, Arce Hecharri J, González Longoria MCB. Estomatología general integral. La Habana: Editorial Ciencias Médicas; 2013.p.364.

5. Pérez Barrero BR, Sánchez Zapata R, Gondín Gener M, Sánchez Garrido

6. AV, Gan Cardero B. Estado periodontal de pacientes atendidos en la Clínica Estomatológica Vista Alegre. MEDISAN . 2012 [citado 5 Jun 2017];16(4). Disponible en: http://scielo.sld.cu/scielo.php?script=sci_arttext\&pid=S1029-

7. $\underline{30192012000400007 \& \operatorname{lng}=\mathrm{es} \& n \mathrm{~nm}=\mathrm{iso} \& \mathrm{tlng}=\mathrm{es}}$

8. Iglesias Berlanga GE, Iglesias Berlanga IJ, Pérez Báez N, González Iglesias A. Estado periodontal en adultos del área Cangre del municipio de Güines. Revista de Ciencias Médicas La 
Habana . 2012 [citado 5 Jun 2017];18(1). Disponible en: http://revcmhabana.sld.cu/index.php/rcmh/article/view/558/html

9. OMS. Promoción de la salud y prevención de enfermedades no transmisibles.2017. http://www.paho.org/ecu/index.php?option=com_content\&view=article\&id=272:promocion-

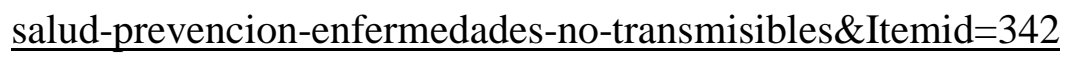

10. Sueiro Sánchez I, Hernández Millán A, Díaz Sosa G, Padilla Gómez E, Díaz Sosa C, Hernández Millán Z. Estado de salud bucal en pacientes discapacitados del área de salud de Espartaco, municipio Palmira. MEDISUR. 2016 [citado 22 Dic 2016]; 14(6). Disponible en: http://scielo.sld.cu/scielo.php?script=sci_arttext\&pid=S1727-897X2016000600007

11. Zamora Tejeda M, Castañeda Deroncelé M, Hechavarría Martínez B. Caracterización clinicoepidemiológica de pacientes especiales con gingivitis crónica. Revista medisan 2017. [citado 22 oct 2017]; 21(10) Disponible en : http://www.medisan.sld.cu/index.php/san/article/view/1594

12. Pérez Barrero Bernardo Ricardo, Rodríguez Mediaceja Gertrudis, Pérez González América, Rodríguez García Beatriz, Paneque Gamboa María Rosa. Principales factores de riesgo de la gingivitis crónica en pacientes de 15 a 34 años. MEDISAN [Internet]. 2009 [citado Oct 28 2017] ; 13( 4 . Disponible en: http://scielo.sld.cu/scielo.php?script=sci_arttext\&pid=S102930192009000400006\&lng=es.

13. Valerazo Bravo TL, Saritama Guarnizo BG. Apiñamiento dental y su relación con el grado de enfermedad periodontal en los estudiantes de 18 a 31 años de edad de la Universidad Nacional de Loja de la modalidad de estudios presencial en el periodo mayo-julio 2014 [citado 22 Dic 2016]. Disponible en: http://dspace.unl.edu.ec/jspui/handle/123456789/18096

14. Zaldívar Bernal HL, Cid Rodríguez MC, Sánchez Gaul JM, Montes de Oca Ramos R. Comportamiento de la gingivitis crónica en adolescentes de la Secundaria Básica Jesús Fernández, Santa Marta, Cárdenas. Rev Med Electron. 2014 [citado 22 Dic 2016]; 6(supl.1). Disponible en: http://scielo.sld.cu/scielo.php.script=sci.arttex\&pid=51684-18242014000700004

15. Gallegos Suquilanda GE. Factores de riesgo socioeconómicos y culturales que influyen en la salud bucal de los niños y adolescentes especiales que acuden al centro de atención y desarrollo "El Ángel". Periodo marzojulio 2015 [citado 5 Dic 2016]. Disponible en: http://.dspace.unl.edu.ec/jspui/bistream/123456789/148121 
16. Cupé-Araujo Ana Cecilia, García-Rupaya Carmen Rosa. Conocimientos de los padres sobre la salud bucal de niños preescolares: desarrollo y validación de un instrumento. Rev. Estomatol. Herediana [Internet]. 2015 Abr [citado 2017 Oct 28] ; 25( 2 ): 112-121. Disponible en: http://www.scielo.org.pe/scielo.php?script=sci_arttext\&pid=S1019$\underline{43552015000200004 \& \operatorname{lng}=\mathrm{es}}$.

17. Chileno Arana M. Relación entre el nivel de conocimiento de los padres sobre salud oral y presencia de caries e higiene oral en escolares con discapacidad. Tesis Para optar el Título Profesional de Cirujano Dentista. 2016. [citado 2017 Oct 28] Disponible en : cybertesis.unmsm.edu.pe/bitstream/cybertesis/5454/1/Chileno_am.pdf

18. Pereira S. Discrasias sanguíneas. Consideraciones generales y Manejo Odontológico. Universidad del Zulia. Facultad de Odontología. Cátedra de Medicina Interna 2003; 185-63.

19. Pérez Requejo JL. Hematología Tomo II. $3^{\text {a }}$ Edición. Ed. Disinlilmed, C.A. Caracas

20. Marchena Rodríguez, L, Fernández Ortega, C M. Atención odontológica a pacientes discapacitados. Revistas europea de odontoestomatologia 2015. [citado 12 oct 2017]. Disponible en : http://www.redoe.com/ver.php?id=219

21. Bécquer Águila BJ, López Díaz Z. Alteraciones de la salud del adulto mayor. En: González Naya G, Montero del Castillo ME, Aldama Bellon Y, Alfaro Mon M, Arce Hecharri J, González Longoria MCB. Estomatología general integral. La Habana: Editorial Ciencias Médicas; 2013.p.364.

22. Pérez Barrero BR, Sánchez Zapata R, Gondín Gener M, Sánchez Garrido

23. AV, Gan Cardero B. Estado periodontal de pacientes atendidos en la Clínica Estomatológica Vista Alegre. MEDISAN . 2012 Abr [citado 5 Jun 2017];16(4). Disponible en: http://scielo.sld.cu/scielo.php?script=sci_arttext\&pid=S1029-

24. $30192012000400007 \& \operatorname{lng}=\mathrm{es} \& n r m=$ iso \& $\operatorname{tn} g=\mathrm{es}$

25. Iglesias Berlanga GE, Iglesias Berlanga IJ, Pérez Báez N, González Iglesias A. Estado periodontal en adultos del área Cangre del municipio de Güines. Revista de Ciencias Médicas La Habana. 2012 [citado 5 Jun 2017];18(1). Disponible en: http://revcmhabana.sld.cu/index.php/rcmh/article/view/558/html

26. OMS. Promoción de la salud y prevención de enfermedades no transmisibles.2017. http://www.paho.org/ecu/index.php?option=com_content $\& v i e w=a r t i c l e \& i d=272:$ promocionsalud-prevencion-enfermedades-no-transmisibles\&Itemid=342 
27. Sueiro Sánchez I, Hernández Millán A, Díaz Sosa G, Padilla Gómez E, Díaz Sosa C, Hernández Millán Z. Estado de salud bucal en pacientes discapacitados del área de salud de Espartaco, municipio Palmira. MEDISUR. 2016 [citado 22 Dic 2016]; 14(6). Disponible en: http://scielo.sld.cu/scielo.php?script=sci_arttext\&pid=S1727-897X2016000600007

28. Zamora Tejeda M, Castañeda Deroncelé M, Hechavarría Martínez B . Caracterización clinicoepidemiológica de pacientes especiales con gingivitis crónica. Revista medisan 2017. [citado 22 oct 2017]; 21(10) Disponible en : http://www.medisan.sld.cu/index.php/san/article/view/1594

29. Pérez Barrero Bernardo Ricardo, Rodríguez Mediaceja Gertrudis, Pérez González América, Rodríguez García Beatriz, Paneque Gamboa María Rosa. Principales factores de riesgo de la gingivitis crónica en pacientes de 15 a 34 años. MEDISAN [Internet]. 2009 [citado Oct 28 2017] ; 13( 4 . Disponible en: http://scielo.sld.cu/scielo.php?script=sci_arttext\&pid=S102930192009000400006\&lng=es.

30. Valerazo Bravo TL, Saritama Guarnizo BG. Apiñamiento dental y su relación con el grado de enfermedad periodontal en los estudiantes de 18 a 31 años de edad de la Universidad Nacional de Loja de la modalidad de estudios presencial en el periodo mayo-julio 2014 [citado 22 Dic 2016]. Disponible en: http://dspace.unl.edu.ec/jspui/handle/123456789/18096

31. Zaldívar Bernal HL, Cid Rodríguez MC, Sánchez Gaul JM, Montes de Oca Ramos R. Comportamiento de la gingivitis crónica en adolescentes de la Secundaria Básica Jesús Fernández, Santa Marta, Cárdenas. Rev Med Electron. 2014 [citado 22 Dic 2016]; 6(supl.1). Disponible en: http://scielo.sld.cu/scielo.php.script=sci.arttex\&pid=51684-18242014000700004

32. Gallegos Suquilanda GE. Factores de riesgo socioeconómicos y culturales que influyen en la salud bucal de los niños y adolescentes especiales que acuden al centro de atención y desarrollo "El Ángel". Periodo marzojulio 2015 [citado 5 Dic 2016]. Disponible en: http://.dspace.unl.edu.ec/jspui/bistream/123456789/148121 